\title{
Un livre documentaire scientifique pour enfants : entre vulgarisation et littérature
}

Daniel Jacobi

\section{(2) OpenEdition}

1 Journals

Édition électronique

URL : http://journals.openedition.org/questionsdecommunication/5729

DOI : 10.4000/questionsdecommunication.5729

ISSN : 2259-8901

Éditeur

Presses universitaires de Lorraine

Édition imprimée

Date de publication : 1 décembre 2003

Pagination : $325-341$

ISSN : 1633-5961

\section{Référence électronique}

Daniel Jacobi, « Un livre documentaire scientifique pour enfants : entre vulgarisation et littérature »,

Questions de communication [En ligne], 4 | 2003, mis en ligne le 23 mai 2012, consulté le 23 avril 2019.

URL : http://journals.openedition.org/questionsdecommunication/5729 ; DOI : 10.4000/

questionsdecommunication.5729 


\section{$>$ NOTES DE RECHERCHE}

DANIEL JACOBI

Laboratoire Culture et communication

Université d'Avignon et des pays du Vaucluse

Daniel.Jacobi@univ-avignon.fr

\section{UN LIVRE DOCUMENTAIRE SCIENTIFIQUE POUR ENFANTS : ENTRE VULGARISATION ET LITTÉRATURE}

Résumé. - Cet article examine un classique de la littérature scientifique documentaire pour enfants : Apoutsiak, le petit flocon de neige, publié par le grand explorateur-ethnographe Paul-Émile Victor dans la collection de L'Atelier du Père Castor de Flammarion, en 1948. Trois approches communicationnelles sont mobilisées pour situer ce chef d'œuvre de littérature enfantine toujours édité, plus de cinquante plus tard : la transposition textuelle et visuelle de l'information scientifique pour un jeune public, la narrativisation du reportage documentaire, et, pour conclure, une discussion du catalogage des genres de la littérature pour enfants.

Mots clés. - Vulgarisation scientifique, documentaire, album pour enfants, récit, ethnographie, Esquimau. 
Dans un tel monde tendu de couleurs, poreux, où à chaque pas tout va se déplacer, l'enfant est accueilli comme un partenaire de jeu.

Benjamin (1972 : 93-94)

L e lundi 10 août 1936, Jean Charcot, capitaine du Pourquoi-Pas?, débarque PaulÉmile Victor au Groënland. Pour lui, avoue-t-il, débute à cet instant : « [ . . . la plus passionnante des vies d'aventures pour la plus passionnante des recherches : la recherche ethnographique » (Victor, $1938: 21$ ). Pour mener plus avant les recherches qu'il conduit sur les Esquimaux, Paul-Émile Victor entreprend de vivre pendant sept mois au sein d'une tribu de vingt-cinq Esquimaux. Et, en particulier, de partager avec eux la longue nuit d'hiver, dans une hutte de six mètres sur cinq. À son retour, il publie Boréal. C'est un livre curieux, pour partie carnet de terrain d'un observateur, pour partie méditation sur la vie sauvage et son rapport avec les sociétés modernes. Paul-Émile Victor souligne que, très volontairement, il n'emporte aucun moyen pour rester informé de ce qui se passe dans le pays civilisé qu'il abandonne ; dans son texte, il ajoutera - rétrospectivement, en une sorte de mise en abyme, comme pour signifier sa préférence pour d'autres valeurs et souligner leur caractère dérisoire - le rappel des faits politiques qu'il a délibérément ignorés durant son enquête de terrain. II écrit : « L'année que je viens de passer au milieu de mes vingt-cinq amis Esquimaux a été une année où j'ai eu le bonheur de n'avoir aucun contact avec l'extérieur [...]. On me demande souvent pourquoi je n'ai pas eu de poste de TSF ; je n'ai pas voulu en avoir, et on comprendra facilement pourquoi, je l'espère » (Victor, 1938 : 29).

En 1948, le chercheur, entré au CNRS, écrit et dessine un livre pour enfants intitulé Apoutsiak, le petit flocon de neige. Ce n'est, pas plus qu'elle ne le serait aujourd'hui, une démarche courante, ou très bien considérée dans un grand organisme de recherche. On soupçonne toujours, dans ce cas, que le chercheur gaspille son temps, ou pire, qu'il n'a pas de vraie recherche à faire. À travers l'histoire d'un enfant esquimau, plusieurs générations de jeunes lecteurs ont pu se familiariser avec les résultats scientifiques des explorations que géographes, médecins et ethnographes accumulèrent au cours $d u X{ }^{e}$ siècle à propos de l'une des dernières portions encore inconnues de la terre : le Pôle Nord. Apoutsiak se présente sous la forme classique d'un récit illustré, destiné à des enfants déjà lecteurs ou peut-être un peu plus âgés. Dès lors que l'on consulte et surtout que l'on lit l'ouvrage, on perçoit nettement deux plans différents : d'un côté, il s'agit bien d'un album pour enfants, aux dessins soignés et à la typographie recherchée ; de l'autre, c'est un documentaire scientifique authentique dessiné et écrit par un scientifique explorateur, un spécialiste déjà renommé au moment où il se lance dans cette entreprise'. C'est ce cas très singulier de communication scientifique que

\footnotetext{
Apoutsiak, le petit flocon de neige, (« Histoire esquimau racontée et illustrée par Paul-Émile Victor », Les albums du Père Castor, Flammarion, 1948) a été, sans cesse republié. Ce n'est sans doute pas un hasard ; les collections de L'atelier du Père Castor sont citées dans le tome 4 de la volumineuse Histoire de l'édition française comme un tournant de l'édition. Paul Faucher, le créateur de cet « atelier », est un éditeur peu ordinaire. Militant de l'éducation nouvelle et partisan des méthodes actives, il a voulu faire du livre et de la lecture un nouvel outil au service d'un projet centré sur l'enfant. Dans cette entreprise, son apport a été d'introduire les innovations typographiques de l'Europe de l'Est, faisant ainsi judicieusement converger l'inventivité graphique et le renouvellement issu des idées nouvelles sur l'éducation.
} 
nous nous proposons d'analyser. Dans un premier temps, nous montrerons le rapport subtil que cet album pour enfants construit entre livre documentaire et recherche scientifique. Puis, nous examinerons comme les procédés de narration le tirent vers la littérature, avant de revenir sur la notion de genre.

\section{Réalisme documentaire et perspective éducative}

\section{Reportage et anthropologie visuelle}

Dès le dessin de couverture, ce qui frappe le lecteur est l'étrangeté et l'exotisme de ce qui lui est montré. Pour la recherche ethnographique comme dans un album pour enfants, le dessin est un registre important. Pour le chercheur, il est un moyen de saisir la réalité à l'égal de la prise de notes sur le cahier de terrain. Dans le livre documentaire, il apporte une grande part de l'information tout en illustrant ce dont on parle. Le chercheur-explorateur-ethnographe a lui-même dessiné chaque illustration de sorte que les dessins constituent un reportage, ou mieux, un documentaire en images signé par l'auteur. En effet, les dessins montrent toutes sortes de détails techniques. Mais, ils ont partout un caractère naïf qui combine une sorte de maladresse à la simplification des formes presque toujours trop arrondies. Cette sobriété permet au dessinateur de mettre en évidence des détails d'une grande précision qui, à cause du dépouillement et de la schématisation, deviennent ainsi très apparents. Les couleurs elles-mêmes, peu nombreuses, sont pastellisées, crayonnées sans à plat, lacunaires, comme esquissées. Les tonalités dominantes sont le blanc et un bleu léger (celui de la mer, du ciel, des ombres de la glace). Les seules tâches vives sont de couleur rouge : comme la viande crue, les pommettes rouges des visages et les bas des femmes. Ces dessins, par-delà leur simplicité, fourmillent de détails et d'informations d'une hallucinante précision : la vareuse porte bébé en peau fourrée et le chignon complexe de la mère ici, la structure du kayak faisant apparaître son mode de fabrication plus loin, les nageoires cousues du phoque, un propulseur incrusté d'os, un oumiak qui, le jour, transporte la famille en mer et, retourné, sert d'abri la nuit et ainsi de suite. Les scènes, elles aussi, esquissent des gestes ou des postures très suggestives et montrent avec une authenticité et une précision, parfois crue, les modes de vie, de travail ou de chasse. Ici, un chasseur pagaie tandis qu'il ramène en remorque le phoque qu'il a harponné ; là, un enfant excite la convoitise de son chien en levant haut un morceau de viande ; ailleurs, le geste précis de la femme qui dépèce un phoque. Mais, le dessin saisit aussi des scènes plus complexes. Les enfants jouent et, pour réaliser ce projet, ils ont transformé en luge des peaux de phoque ; quand ils ont grandi, ils ne couchent plus près de leur mère ; enfin, un grand dessin révèle l'incroyable bric-à-brac et 
le désordre des attitudes de tous les personnages qui se trouvent, pour la longue nuit d'hiver, dans la grande hutte collective où les corps se dénudent, dans la tiédeur des lampes à huile pour cuisiner².

En examinant les relations textes-images, on remarque vite que le dessin apporte un lot d'informations et de détails. On est loin du classicisme de l'album illustré où chaque image renvoie à un texte pauvre ou pléonastique. En tout cas, beaucoup de ces images-documents ne sont pas en phase avec le récit ou la chronologie de l'exposé. Certains détails apparaissent dans les dessins, bien avant que le texte n'y fasse explicitement allusion. Puis, ils reviennent au moment où ils interviennent dans le récit ou le contexte et sont éventuellement repris, sans raison apparente. Du coup, ils constituent à la fois un motif et, par leur reprise, une sorte de rime visuelle. Ainsi, des éléments comme la neige, la mer, la glace, la peau du phoque sont-ils quasi inamovibles et, de la sorte, présents dans le dessin à chaque page. Cette répétition installe et active un climat, une atmosphère permanente qui s'impose au lecteur. Par exemple, le dessin de l'Esquimau en chasseur sur son kayak (p. 2) est une reprise simplifiée du dessin quasi encyclopédique de la couverture du livre. D'une précision technique insolite, l'image-accroche du livre condense une série d'informations, dignes de croquis, que l'ethnographe consigne en tant que relevés d'observation sur le terrain. Chaque détail est représenté minutieusement pour faire apparaître les matériaux, la fonction et les procédés de fixation sur le pont du kayak dont on aperçoit l'armature et le revêtement en peau de phoque. Le dessin est repris plus loin (p. 20) : il permet de décrire l'armement du kayak à l'arrêt sur lequel le chasseur a pris place en fumant sa pipe. On voit nettement le rectangle blanc de tissu, le tripode, le ballon orangé et surtout le harpon posé à côté de l'Esquimau. Ces trois détails sont repris à la page suivante (p. 22), celle d'un retour de chasse. On remarque alors le cordage qui relie le harpon au phoque remorqué et la sphère orange qui sert de repère sur l'eau. Le texte identifie ici les objets et la technique de harponnage (p. 22) :

Son harpon est posé à côté de lui.

La boule jaune est un flotteur

qui empêche le phoque de se sauver

une fois qu'il est harponné.

Devant Apoutsiak, il y a un petit trépied sur lequel est enroulée la courroie

qui relie le flotteur à la pointe du harpon. Et tout à fait à l'avant du kayak,

il y a un petit écran blanc

derrière lequel Apoutsiak se cache

pour que les animaux qu'il chasse ne le voient pas.

${ }^{2}$ On se souvient que P. Bourdieu (celui de la première période, ethnologue en Kabylie) avait, en 196364, dressé des plans d'habitation pour son étude : La maison ou le monde renversé (Bourdieu, 1972). 
On remarque le parfait travail de vignetage encyclopédique qui assure la correspondance descriptive texte-image. Pour raffiner le tout, le graphiste utilise une ligne non justifiée à droite qui varie de $3,5 \mathrm{~cm}$ à près de $9 \mathrm{~cm}$ pour la plus longue. Un peu comme s'il avait voulu habiller l'image du kayak ou faire jouer à la ligne un rôle de légendage. En fait, chaque retour à la ligne résulte d'une décision : passer à la ligne correspond systématiquement au saut d'une unité d'information à l'autre. Chaque ligne ménage ainsi comme un effet de surprise pour le lecteur, tout en l'aidant à structurer les unités de sens. Et la ligne la plus longue correspond au dévoilement d'une énigme : celle du petit carré blanc placé à l'avant du kayak pour camoufler l'Esquimau. Le même procédé de répétition permet au lecteur de se situer dans le paysage. Dès la page 2 , on remarque un grand iceberg de forme érigée surprenante (sa silhouette évoque la célèbre falaise d'Étretat), au milieu d'un fjord, dans un paysage d'été. On retrouve, en arrière plan du même paysage, l'hiver (le fjord devenu blanc est gelé), le même iceberg (p. I3). Et enfin, sur la double page (pp. 24-25), l'enfant pourra comparer la forme de cet iceberg dont la silhouette change en fondant l'été. À cet instant, le texte apporte la précision suivante : « Sur le fjord, un grand iceberg est venu s'arrêter, car son pied touche le fond ». Manière, on le voit, de décrire et d'évoquer le contraste des saisons et l'importance des transformations du paysage qui en résultent.

\section{Le dédoublement énonciatif}

Comme pour confirmer le statut de documentaire, le texte de l'album est découpé en deux registres. Sans aucune difficulté (taille des caractères et emplacement), le lecteur repère cette double textualité. D'une part, un texte central qui constitue le fil de l'histoire. D'autre part, les sortes de notices explicatives, en marge, qui apparaissent comme un texte secondaire, périphérique. Outre les marques d'édition qui les distinguent sans ambiguïté, leurs formes énonciatives séparent nettement les deux types de textes. L'un, le texte principal, est un récit écrit à l'imparfait. II a pour unique héros un jeune Esquimau. II est écrit à la troisième personne du singulier (《Apoutsiak », « il »). Le second texte, rédigé au présent, est un commentaire explicite des vignettes, sous forme d'une description plus impersonnelle. Occurrent à chaque double page, il débute par une marque déictique appuyée et adressée directement à l'enfant lecteur : « Voici la maman..., Ceci est un phoque..., Quant à ça, c'est une lampe..., Tu vois sur cette image ». II correspond à un commentaire technique précis et documenté des modes de vie, des façons de faire et de l'outillage traditionnel mobilisé pour survivre dans un milieu naturel, sauvage, singulier et hostile. Truffé d'incitations à observer, son tutoiement souligne que l'auteur s'adresse effectivement à l'enfant pour guider son regard sur certains des détails des dessins disséminés sur les pages. Comme pour soutenir et relancer l'attention, dans presque chacun de ces énoncés, Paul-Émile Victor écrit : «Tu vois, vois-tu, tu peux voir, tu reconnais, remarque... ». 
Le texte peut aussi inviter l'enfant lecteur, presque de façon injonctive, à organiser sa prise d'information avec, par exemple, ce futur d'obligation programmatique («Au dos de la couverture, tu verras que ce pays... »). Cependant, on note que la description est enrichie de tout un répertoire de reformulations au caractère très nettement métalinguistique. Ainsi, le terme spécialisé « icebergs » est paraphrasé par expansion dans la relative cooccurrente : «Sur la mer flottent de beaux icebergs, qui [sont] d'énormes morceaux de glace ». Mais les formes de ces textes secondaires sont en réalité assez souples. Outre leur rôle de monstration et de reformulation, les petits textes revêtent aussi des tours explicatifs : « Ses nageoires sont cousues ensemble pour éviter qu'elles freinent en traînant dans l'eau » (p. 4) ; « ...des morceaux de graisse de phoque qui donnent de l'huile » (p. 4). II lui demande, un peu comme un maître qui vérifierait si le lecteur a été attentif, de se rappeler (p. 26) d'une information déjà donnée quelques pages auparavant: « Dans le ciel, il y a trois soleils cette fois-ci. Te souviens-tu du nom de ce phénomène ? »». Et même, il lui fait parfois part d'une sorte de confidence : « C'est magnifique ['aurore boréale]! Tu sais : chaque fois qu'on en voit une, on reste tout étonné, comme toi quand tu as vu un arc-en-ciel pour la première fois » (p. 29).

Pour résumer le rôle de ce texte périphérique, on voit qu'il apporte une riche et dense information scientifique et technique tout en incitant l'enfant lecteur, dans une adresse personnalisée sans cesse réitérée, à observer activement et à retenir les informations ainsi exhibées. Le contrat de communication instauré par ce dispositif scriptovisuel, déjà vieux d'un demi-siècle, apparaît, de la sorte, d'une remarquable modernité : il préfigure la page du magazine illustré qui ne verra le jour, en France, qu'une vingtaine d'années plus tard. Et il instaure ce rapprochement entre le texte documentaire et la posture de reconnaissance mi-encyclopédique, mi-studieuse qui convient à cette catégorie de discours.

\section{Du document au récit : la narration en actes}

Le texte principal de l'album est facile à repérer par sa centralité et la taille supérieure des caractères. II est assez bref et apparaît régulièrement sur chacune des doubles pages du cahier ouvert. II est écrit à l'aide de phrases courtes ou bien structurées par la ponctuation. Ce texte lie fortement les pages de l'album les unes aux autres : il gère et tient la continuité du livre. En même temps qu'il correspond toujours à l'image principale de la page, il porte la tension narrative, faible mais perceptible, celle du héros qui grandit, devient homme et père, puis vieillit et meurt. Au sens le plus strict, le récit d'Apoutsiak est ce que Mikhail Bakhtine a appelé judicieusement un chronotope (1978 : 238), c'est-à-dire un procédé spatio-temporel de mise en fiction. En dépit de sa simplicité, cette structure est évidemment un procédé efficace. En faisant mine de satisfaire l'une des exigences du genre album pour enfants (celle 
de conter une histoire, de narrer des aventures), il permet au chercheur de condenser une série d'informations scientifiques selon un principe diachronique élémentaire. Le plan de l'enquête ethnographique sur les différents membres de la tribu et leur mode de vie en société est transposé selon un principe de succession linéaire et les relevés de terrain sur les façons de faire des différents groupes d'âge sont attribués à un seul actant (fictif) qui devient l'unique héros de l'ouvrage. L'enquête ethnographique procède systématiquement par des observations distinctes des membres de la tribu et des différentes facettes de l'organisation sociale : les enfants, la maternité, les jeux ; l'entrée dans l'âge adulte, la pêche, la chasse ; les vêtements, la nourriture et le mariage ; les croyances, la vieillesse et la mort. Au contraire, le principe diégétique permet de rassembler toutes les observations en une chronologie personnalisée et spectaculaire. En tournant les pages de l'album et en découvrant le récit d'Apoutsiak, le lecteur explore assez systématiquement les différentes facettes d'une vie sauvage authentique. Paul-Émile Victor prête, au seul Apoutsiak, tous les caractères prototypiques de l'Esquimau idéal qu'il a collectés tout au long de sa méticuleuse série d'observations authentiques. Pour ancrer le récit dans sa grammaire de texte, le scripteur, plus ou moins intuitivement, mobilise un certain nombre de caractéristiques propres au genre. Nous en analyserons trois : la gestion des temps verbaux, la structure du découpage et la mise en texte.

\section{Imparfait et présent}

Le texte d'enquête et les notes prises, au jour le jour, sont - on peut le supposer - écrites au présent. L'enquêteur est un témoin, un observateur qui consigne chaque jour ce qu'il observe. Le temps de l'enquête et le temps vécu coïncident. Le chercheur note pour lui, comme pour fixer à l'état brut des tableaux de vie ou des scènes de la vie qu'il partage, immergé dans le monde qu'il veut observer et découvrir. Devenu écrivant (comme l'a dit lucidement Roland Barthes dans un entretien télévisé) pour d'autres spécialistes ou écrivains, il pourrait rapporter la vie des Esquimaux au présent : un peu comme un journaliste qui, pour associer le lecteur du fait divers à l'action, lui fait revivre la scène de l'action comme si cette dernière se déroulait sous ses yeux (Petitjean, 1987). Pourtant, dans l'album, le récit est écrit à l'imparfait de l'indicatif (et non, comme il est dit-on de règle pour les contes, au passé simple). En installant tout le récit de vie à l'imparfait, le scripteur satisfait l'exigence élémentaire qui conduit à situer l'action dans l'accompli. L'hiver qu'il a passé au Groënland est déjà vieux de douze ans au moment où il écrit le livre pour enfants. Mais, le choix de l'imparfait offre un autre avantage. Rappelons que l'imparfait est le temps du passé qui traduit une action non achevée (ou non accomplie). On le qualifie habituellement de présent en cours dans le passé. Sans problème, il convient donc pour commenter la vie du jeune Esquimau et le fil de son existence que le lecteur découvre et suit en tournant les pages. De 
plus, l'imparfait permet de situer l'action dans une période réelle sans en préciser les limites. Ainsi, la vie des Esquimaux était-elle il y a déjà longtemps ; et elle « est encore ainsi » au moment où le lecteur la découvre. La maîtrise des temps verbaux est manifeste quand la stabilité de l'imparfait est brusquement mise à mal tout près de la fin du livre (p. 27) :

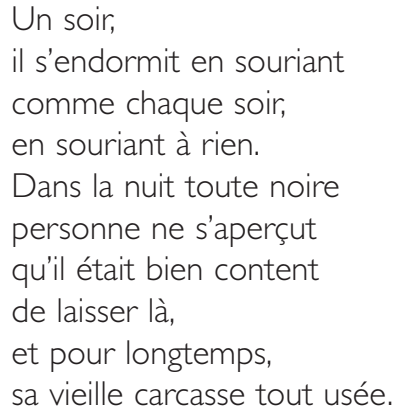

L'apparition du passé simple fait basculer le texte dans le temps du récit, c'est-àdire du passé révolu. Mais l'irruption du passé simple permet aussi de détacher cet événement essentiel (la mort d'Apoutsiak et son arrivée au paradis) d'une situation jusque-là stable et continue. II montre la soudaineté de cet événement : ce passésimple souligne que la mort, nette et implacable, survient sèchement. Pourtant, heureuse résolution, le passé simple précède l'arrivée du présent pour la chute du récit, à la dernière page du livre : « Et maintenant, Petit flocon de neige est heureux... ». L'occurrence du présent (et non pas du futur) correspond à l'intemporalité, à une sorte d'éternité : sans doute au moment où le lecteur tourne la dernière page du livre, peut-il espérer que cet Esquimau-là (Apoutsiak) est encore dans le pays où (semble-t-il), on vit toujours heureux...

\section{Découpage et scénarisation d'une histoire de vie}

Le récit correspond strictement à la vie d'Apoutsiak. Elle est rythmée par une série de tranches de vie, la naissance, à cinq ans (p. 6), à dix ans (p. 12), à vingt ans (p. 18), enfin à cinquante ans (p. 27). En somme, une décennie couvre trois pages de l'album. De la naissance à cinq ans, il est dans la douceur (de la mère) ; de cinq à dix ans, période du jeu et de l'apprentissage, il est impatient ; dès dix ans, il est équipé comme un adulte et il est écarté du monde des enfants ; à vingt ans, il a une femme et un fils et, peu après, chasseur hardi et fort, il est le chef d'une famille élargie ; à cinquante ans, il est vieux, au bout de sa vie, et meurt en souriant. À chaque étape textuelle de la vie, correspond un portrait d'Apoutsiak qui vieillit ainsi à vue d'œil. À vingt ans, on aperçoit déjà ses rides ; et, à cinquante, assis et tassé sur un banc, les cheveux blancs, il peine à mâcher la viande de phoque. Apoutsiak est un représentant prototypique d'une civilisation ancienne et traditionnelle pour laquelle le rapport avec la nature est essentiel. L'Esquimau, sa famille ou sa tribu luttent et rusent pour survivre de façon ingénieuse dans un 
milieu hostile au point que la reproduction des générations pourrait y devenir problématique. Apoutsiak reçoit de ses parents une culture technique raffinée qu'il se dépêche de transmettre à ses enfants avant de mourir, déjà vieux, à peine cinquante ans plus tard. De plus, le récit constitue un texte littéraire soigné qui évoque le poème, aussi bien par sa disposition sur la page, que par ses sonorités et les multiples allitérations qu'on y entend. L'auteur semble avoir ajouté une dimension poétique à cette sorte de prosopopée de la vie sauvage. Par exemple, une sorte de clin d'œil métalinguistique, drôle et inattendu, revient à cinq reprises selon une forme plus ou moins ressemblante. Voici la première de ces occurrences : «Puis il s'endormait, souriant aux anges (aux anges esquimaux, naturellement) » (p. 5). L'auteur revient sur son texte pour, entre parenthèses, faire remarquer au lecteur sa méprise qui, ainsi soulignée, se transforme en un effet délibéré. II fait comme s'il oubliait que l'enfant esquimau n'était pas un enfant semblable au lecteur alors que, à l'évidence, il n'appartient pas à la même civilisation. De façon appuyée, il souligne combien le petit Esquimau est un enfant qui ressemble à l'enfant lecteur. Par leur répétition, ces parenthèses métalinguistiques, sortes de discours dans le discours sur le discours, sonnent comme des ritournelles (ou des refrains), telles celles que les conteurs utilisent pour ponctuer le cours du récit au grand plaisir de leur auditoire.

\section{La littératuralisation des énoncés}

Après avoir construit et installé la double textualité qui structure la page entre centre et périphérie, l'auteur enfreint la règle qu'il s'est lui-même imposée. En effet, il n'est pas rare que le texte secondaire (que l'auteur comme l'éditeur semblent avoir institué comme strictement documentaire) complète ou ajoute au récit principal, des épisodes périphériques. Ainsi, Apoutsiak suit-il son fils au loin qui, avec son traîneau, revient de la chasse au phoque et dételle déjà les chiens (p. 25). Ailleurs, et cela est encore plus inattendu, l'auteur instaure une sorte de complicité avec son lecteur. II l'interpelle directement (p. 13) et implique le héros dans le commentaire : «Aurais-tu peur, toi, d'un tel masque? En tous cas les enfants esquimaux en ont généralement peur ; mais pas Apoutsiak... ». Plus loin, il fait un clin d'œil au lecteur, une sorte de farce amusante (p. 24), en expliquant malicieusement entre parenthèses : « (il n'y a personne sur le dessin, parce que tout le monde est allé cueillir des myrtilles de l'autre côté de l'eau) ». Ce tour est plus complexe qu'il n'y parait. En faisant mine d'expliquer pourquoi les Esquimaux sont absents de l'image, le scripteur fait volte-face et dévoile brutalement une machinerie qui était non dite et sans aucune justification. Un peu comme si la main qui dessine était devenue étrangère et que le scripteur critiquait son double, le dessinateur. On se souvient que les cartoons de Tex Avery ont parfois usé du même procédé pour casser et dédramatiser des fuites éperdues : le film s'arrête, le crayon rejoint le dessin et la voix du dessinateur réalisateur commente froidement l'interruption et remplace celle de son héros. 


\section{Quête ethnographique de la vie sauvage ou récit édifiant pour enfants : les ambiguïtés de la catégorisation en genres}

En somme, dans ce livre pour enfants, l'histoire singulière du héros Apoutsiak fournit une trame narrative (celle de sa vie), puis s'exemplifie en celle d'un Esquimau encore relativement préservé de la société industrielle, avant de déboucher sur une réflexion anthropologique allant, de l'étude fine des rapports d'une société traditionnelle avec le milieu dans lequel elle vit, jusqu'à celle de la complétude d'une civilisation esquimau ancestrale et fortement menacée. Propos ambitieux, on le voit, qui pose la question du genre. On devine qu'Apoutsiak est bien plus qu'un « album pour enfants » qui est la catégorie officielle à laquelle, de facto, il appartient. Publié et sans cesse republié par Flammarion, il est officiellement considéré, par toutes les bibliothèques et autres Centres de documentation et d'information (CDI), comme un livre illustré pour enfants. Mais, c'est d'évidence aussi un ouvrage documentaire (et de première main), ou un livre de vulgarisation (et pas de n'importe quelle vulgarisation, mais de celle faite par le chercheur lui-même).

\section{La nébuleuse de l'édition pour enfants}

Il y a lieu de s'interroger sur la catégorisation des genres comme sur celle des lecteurs. Le domaine du livre pour la jeunesse est lui-même gouverné par la tyrannie des tranches d'âge. À grands coups de conseils, voire de consignes imprimées sur la quatrième de couverture, on conseille à l'acheteur de préférer un ouvrage dûment édité pour l'âge du lecteur auquel on offrira son devoir-lire ${ }^{3}$. Considérer un document comme étant « pour enfants » produit un effet de ségrégation : en choisissant une catégorie exclusive de lecteurs, on écarte les autres ou, pire, on déconsidère l'envie de lire ce genre d'ouvrages ainsi relégués dans un sous-univers de la littérature. C'est le souci de précision ethnographique (il ne quitte pas un instant l'auteur) qui fait rapidement douter du caractère enfantin. Cela transparaît surtout dans le texte secondaire périphérique (descriptif ou explicatif) qui incite le lecteur à observer en détail les dessins. Mais, l'auteur ne répugne pas à glisser des informations de ce type dans le texte du récit (p. 16), quand Apoutsiak quitte la proximité de sa mère, la nuit, pour aller dormir dans la hutte collective « près de la fenêtre avec les grands ». Ou encore quand Apoutsiak atteint sa cinquantième année : « II ne chassait plus, mais ses fils et ses gendres et ses filles et ses brus s'ingéniaient à lui rendre la vie plus douce et plus belle » (p. 27). Indication qui, au passage, révèle que le chef de famille, devenu vieux, est pris en

\footnotetext{
${ }^{3}$ Sur cette question, on peut se référer à l'ouvrage de J.-C. Chamboredon et J.-L. Fabiani (1977). Le livre, les illustrations et le texte lu à voix haute, sont le support de tout un riche travail de médiation et d'interactions (cf. Singly de, 1993 ; Chartier, Hébrard, 1989).
} 
charge par ses enfants. Mais, c'est surtout du point de vue de l'iconographie que le travail de l'ethnographe, devenu dessinateur d'un livre pour enfants, est révélateur. Sans rien ôter de la précision des dessins de terrain qui sont des sortes de prises de notes visuelles, l'auteur contextualise et humanise les croquis secs et rapides faits sur place au moment de l'observation. II les met en scène dans des tableaux complexes où apparaissent, non seulement le paysage et ses couleurs, mais aussi les outils et les techniques manipulés par les personnages humains, parfois euxmêmes en interaction avec d'autres acteurs. Ainsi, le considérer comme un « album pour enfants », ou comme relevant du genre « documentaire scientifique », est-il évidemment très réducteur. Deux pistes différentes méritent d'être discutées : en quoi s'agit-il d'un document, d'une part, pourquoi relève-t-il de la culture scientifique, d'autre part?

\section{L'orientation scientifique}

II est tentant de considérer que ce livre relève de la « culture scientifique et technique ». On sait cependant que cette notion est ambiguë. La question de savoir si un document ou une œuvre est « culturelle » (ou ne l'est pas) est, à certains égards, sans objet. Le débat sur ce sujet, ouvert dès les années cinquante par Charles Percy Snow (1968), a été plus obscurci que déplacé avec la popularisation (relative) de la notion de « culture scientifique », après les États généraux de la recherche en 1981. La structure du livre, nous l'avons souligné, apparait comme un croisement ou une superposition de deux axes : la chronologie des âges de la vie (celle des Esquimaux dont le tempo, on l'a vu, est plus rapide que celle d'un européen) et le découpage strict des ethnographes qui, pour décrire et analyser exhaustivement une société inconnue, distinguent des plans d'observations focalisés et distincts. Un peu comme les manuels enseignent aux enquêteurs de terrain comment recueillir méthodiquement toutes les caractéristiques d'une tribu indigène en distinguant sa morphologie sociale (géographie, démographie), sa physiologie (techniques, esthétique, jeux, éducation, droit, économie, religion...) et les phénomènes généraux (langue, échanges...). Pour mieux saisir en quoi l'ouvrage est scientifique, il convient de faire un détour par l'épistémologie de la discipline. Par exemple, au Manuel de Marcel Mauss (1947) qui résume les cours qu'il a professés à Paris, entre 1935 et 1939, il est évident que la structure de l'album pour enfants s'inspire directement du travail scientifique de terrain qui transparait dans chacune des pages, aussi bien dans le texte que dans l'illustration. Par exemple, pour la chasse, Marcel Mauss préconise de ne pas la considérer comme une activité générique mais de distinguer, au contraire, les différentes sortes de chasse selon le gibier recherché : « La chasse peut s'étudier de deux manières principales : selon l'arme employée, selon le gibier poursuivi (armes, techniques, moment de l'année, etc.). L'individu ne va pas "à la chasse", il va à la chasse au lièvre ; et non pas à la chasse au lièvre mais à la chasse de tel lièvre qu'il connaît bien ; il faut donc classer par gens qui chassent, gibier chassé et instrument avec lequel il est chassé » (Mauss, 1947 : 58). 
C'est exactement ce que fait Paul-Émile Victor en distinguant la chasse au phoque (p. 20 ; p. 22) de la chasse à l'ours (p. 23), ou encore de celle du narval (p. 26). Le phoque est poursuivi en kayak et chassé à l'aide d'un harpon. L'Esquimau embusqué chasse l'ours blanc au fusil. Quant au narval, dont la chasse est très dangereuse, on le tue depuis un kayak, au harpon, car « si on le tirait au fusil, le narval tué coulerait aussitôt » (p. 26). En examinant, d'une part, chacune des illustrations, et d'autre part, les deux catégories de textes, on vérifie que la qualité et la méticulosité des informations ethnologiques et anthropologiques que Paul-Émile Victor propose sont non seulement réelles et authentiques mais aussi racontées, jusque dans le moindre détail, avec un grand souci de vérité et d'exactitude ${ }^{4}$. Les faits sont rapportés sans fard. Par exemple, le chien tellement utile et admiré quand il travaille, peut importuner la femme qui cuisine dans la hutte : « [...] et toute cette nourriture attire les chiens, que la maman d'Apoutsiak chasse à coups de pieds » (p. 10). Considérer la science comme une sorte de sous-secteur, qui serait rangé à part et devrait être examiné d'un œil différent, revient sans doute, de la plus sûre façon, à la reléguer définitivement hors de ce qui serait la « vraie »culture. Si on considère Apoutsiak comme un vrai livre, quel est donc le genre littéraire auquel il appartient ? Au plan de la littérature, on pense évidemment au roman d'aventures, et à des livres comme Le dernier des Mohicans de Fenimore Cooper ou à Croc Blanc de Jack London. Rappelons que tous deux ont été aussi souvent publiés dans des collections littéraires que considérés comme des œuvres « pour la jeunesse ». La qualité de ces romans provient, entre autres facteurs, de ce que l'un comme l'autre de ces romans, a su remarquablement intégrer les descriptions des explorateurs ethnographes dans les aventures de leurs héros. Est-ce que, démarche inverse de celle du romancier, Paul-Émile Victor n'est pas parvenu à transformer un projet de divulgation scientifique en un pur chef d'œuvre de la littérature?

\section{La piste documentaire}

Explorons l'autre piste, celle du « parti pris du document » (Chevrier, Roussin, 2001 ). Nous avons déjà noté que la matière narrative d'Apoutsiak semble tout entière constituée, de façon homogène, d'observations ethnographiques scrupuleuses et authentiques faites par l'auteur en tant qu'observateur ou témoin impliqué. On serait ainsi devant une œuvre de littérature-vérité (pour paraphraser la notion de cinéma-vérité). Cependant, ne négligeons pas la part d'inventivité et de reconstruction que représente toute fictionnalisation de la réalité. Évidemment, les mêmes matériaux authentiques, saisis par deux auteurs

\footnotetext{
${ }^{4}$ II est difficile de se prononcer sur les qualités scientifiques de P.-É.Victor qui est davantage connu comme explorateur que chercheur. Malaurie, le célèbre spécialiste français des déserts glacés et des Esquimaux, ne le cite pas dans sa galerie des grands explorateurs du Pôle. II rappelle qu'il a été plutôt un organisateur, « un maître de l'entreprise », en créant une agence des expéditions polaires françaises sous le nom de Missions Paul-Émile Victor (Malaurie, 1990 : 369).
} 
différents, ne donnent pas lieu à la production d'œuvres identiques, du point de vue de la trame narrative ou de la qualité de leur présentation (au sens de leur contextualisation). Au moment où l'auteur enregistre les moments de vie (c'està-dire les documents proprement dits), il a nécessairement en tête un projet de communication et de reconstruction de la réalité. Dans ce qu'il voit, décrit ou dessine, il filtre et sélectionne les clichés les plus à même de traduire ce qu'il ressent et veut communiquer à d'autres. Si le souci de précision et la volonté de rester aussi proche que possible d'une ethnographie de terrain confirme la piste du documentaire, on pourrait s'étonner du jeu, que nous avons plusieurs fois mentionné, qui propose un parallèle entre cette civilisation esquimau, indigène et primitive, et la nôtre. Certains éducateurs y ont vu, non sans raison, la préoccupation de favoriser en permanence l'identification du petit lecteur avec le jeune Esquimau. Manière, en quelque sorte, d'éradiquer toute tentation d'interprétation raciste qui rabaisserait l'Inuit sauvage et primitif. Mais, c'est oublier bien vite la réalité colonisatrice. L'année où Paul-Émile Victor, lors d'une de ses premières expéditions polaires, hiverne avec les Esquimaux, il n'ignore pas que Canadiens et Danois ont depuis longtemps entrepris de nouer des relations commerciales avec les peuples du grand nord; ni que les missionnaires ont commencé la christianisation des peuples esquimaux, et ce, dès le début du XIXe siècle. L'ethnographe qui, en 1936, s'intéresse aux mythes et religions comme toute enquête systématique l'incite à le faire - recueille évidemment une superposition des anciennes croyances chamanistes avec les topiques chrétiens. Les masques de sorcellerie ne font plus peur qu'aux enfants et le « marchand de sable » flotte au-dessus de l'enfant qui s'endort en souriant (p. 9). En revanche, après leur mort, les Esquimaux vont, eux aussi, dorénavant, au paradis. L'univers de la nuit qui est très présent et récurrent (p. 5 avec les anges ; puis p. 9 avec le marchand de sable) et le paradis final sont dessinés par Paul-Émile Victor avec des moyens sémiotiques voisins. D'abord, il les associe aux astres et aux phénomènes célestes (étoiles, parhélie, aurore boréale), bref à ce qui élève les yeux des hommes vers l'espace et l'au-delà. Ensuite, il colore le ciel en foncé et y dessine de brillantes étoiles, tracées naïvement à l'aide de petits traits croisés. Au besoin, il agrémente le fond de ces scènes dessinées d'une sorte d'ondulation en semis, comme si l'obscurité vibrait. Enfin, pour représenter un paradis, étrangement semblable aux scènes terrestres, il se contente de placer une étoile au-dessus de la tête de chacun des actants, qu'il s'agisse des Esquimaux, du phoque ou de l'ours blanc. La présence insistante et appuyée du « paradis » (des anges, ou de l'âme qui s'échappe du corps, p. 28) semble une sorte de bizarrerie dans un livre qui, à bien des égards, est aussi un reportage scientifique précis et scrupuleux sur des pratiques ancestrales. On se souvient que l'arrivée au paradis est dramatisée par l'irruption de verbes au passé simple (en opposition à l'imparfait jusque-là employé seul) et que la dernière phrase du livre, inscrite en haut de la dernière image, au présent, est : « Et maintenant Petit flocon de neige est heureux comme on ne peut l'être qu'au paradis... » (p. 32). L'image montre Apoutsiak assis devant sa hutte face à une femme vue de dos. Une autre femme (avec un bébé dans le capuchon 
de sa veste) est à sa droite et un enfant, déjà grand, joue à sa gauche. Au loin, on aperçoit un phoque et au premier plan un ours blanc. La scène est débonnaire, les animaux pacifiques. Hommes et bêtes sont surmontés d'une petite étoile, au-dessus de leur tête, qui seule singularise le paradis des autres scènes réelles, très ressemblantes à celle-ci. Pourquoi un telle fin ? La chute conclusive de l'histoire serait-elle destinée à constituer une fin heureuse pour rasséréner, selon un stéréotype convenu, le jeune lecteur? Une tout autre interprétation peut être esquissée. L'ethnographe, immergé dans la tribu dont il a appris la langue et partagé les joies et les peines, a aussi découvert les misères et les difficultés que des hommes, dominés et déjà fortement dépendants des autres pays développés, affrontent. À côté des outils et instruments traditionnels fabriqués avec les matériaux locaux (kayak, propulseur, harpon...), on voit partout les objets manufacturés (fusil, couteau, mais aussi tabac) échangés au comptoir danois contre des fourrures et des peaux. Ainsi, sur le grand oumiak qui transporte la famille élargie (p. 20), aperçoit-on, à l'avant comme à l'arrière du bateau, des rouleaux de peaux de phoques qu' « Apoutsiak ira échanger au comptoir danois contre les chandails de laine, du tissu, du riz, du sucre ».

Si donc il est manifeste que l'album pour enfants est directement nourri des résultats de la recherche et qu'il en représente une sorte de travail de transposition en vue de sa diffusion, en direction des enfants européens, il en conserve aussi une dimension éthique implicite correspondant à la position quasi militante du chercheur. Évidemment, cette clé de lecture conduit l'observateur à reconsidérer totalement l'analyse d'Apoutsiak, qu'il s'agisse du texte ou de l'iconographie. La vie dans les déserts glacés est dure, difficile, la survie même de ce type de civilisation, tout à fait hypothétique au milieu du $X X^{e}$ siècle. Paul-Émile Victor sait pertinemment que l'existence des Esquimaux et d'un mode de vie en harmonie avec un espace immense, encore préservé, est plus que compromise. Les Esquimaux du Groënland, et c'est probablement pour cela que l'exploration a été décidée, sont condamnés à disparaître. En somme, comment les sauver? Quel espoir, autre qu'imaginaire, de les voir survivre au rouleau compresseur de l'uniformisation des modes de vie urbains civilisés ? En plaçant au présent de l'indicatif (présent intemporel ? présent d'éternité ?) son héros Apoutsiak au sein d'un paradis, Paul-Émile Victor ne tente-t-il pas d'élever cette civilisation au panthéon archétypique des grandes civilisations?

\section{Conclusion}

En 1948, Paul Faucher, qui va contribuer à réveiller le monde de l'édition française, rencontre Paul-Émile Victor. Grand explorateur déjà célèbre, il est à la fois un auteur très bien informé par ses voyages et ses recherches et un dessinateur talentueux, disposant de carnets de croquis quasi inédits. Bouleversé 
par l'expérience humaine dont il rêvait, et qu'il a enfin vécu, il a manifestement envie de s'adresser aux enfants. II prend très au sérieux ce projet : il fournit à ses lecteurs des informations dépourvues de mièvrerie, crues, précises, vérifiables à chaque page et confirmées dans tout le discours. II écrit un texte, beau et poétique, retrouvant intuitivement le charme de la tradition du conteur, ses répétitions et ses allitérations. On sent aussi combien, du début jusqu'à la fin, il considère les enfants comme de vrais lecteurs, des lecteurs attentifs et exigeants. Ni mièvrerie, ni réalité arrangée, ni censure : les chasseurs tuent les animaux qu'ils guettent à l'affût, les chien importuns sont écartés d'un coup de pied et la réalité des éléments imposent sa loi implacable. Paul Faucher et ses collaborateurs, par une mise en page impeccable, soulignent et renforcent le projet de communication. Les choix typographiques, tous légitimes, les légères transformations poétiques et calligraphiques qu'ils ajoutent, sont d'un raffinement rare et presque invisible car toujours au service du projet de communication. Et, il n'est pas certain que la collection du Père Castor, pourtant réputée, soit parvenue à chaque titre à réussir un tel équilibre. Dans la mise en page, alternent des dessins pleine page (bords perdus, comme si l'espace du paysage ou de la scène débordait l'aire scripturale) avec d'autres dont les contours arrondis et estompés évoquent des images souvenirs déjà enfouies dans la mémoire. En tournant les pages du livre, on fait inexorablement défiler le fil du temps, des années qui passent, du héros qui grandit et vieillit. Et pourtant, en ouvrant le cahier, on va de surprise en surprise, de la tendresse à l'action, du sommeil et du rêve à l'impatience de grandir, du réel du jeu des enfants aux occupations des adultes, de l'été à l'hiver, de la nuit au jour, de la chaude solidarité du groupe à la solitude du départ vers l'au-delà.

Pourquoi pourtant, par-devers le soin et l'inventivité graphique de sa réalisation, peut-on considérer Apoutsiak comme une œuvre authentique de littérature? Aux grandes qualités rédactionnelles et iconographiques de cet album pour enfants, s'ajoute évidemment ce qui relève à la fois de l'intérêt du thème anthropologique et de l'engagement profond du chercheur. On sait que la découverte et l'exploration ont été, tout au cours du XIX $X^{e}$ siècle, l'un des thèmes les plus récurrents, mais aussi des plus populaires de la littérature de vulgarisation. La revue L'Illustration a fréquemment publié des récits reportages sur les peuples sauvages rapportés de lointaines contrées. La recherche de l'homme des origines ou celle d'une région géographique lointaine totalement oubliée dans laquelle aurait été préservée, hors du temps, une société authentiquement naturelle est certes une quête fantasmatique (Cohen, 1999). Mais, elle a mobilisé (et mobilise encore) non seulement des chercheurs et des spécialistes, mais aussi tous ceux qui recherchent naïvement le dépaysement et l'exotisme. On peut évidemment discuter la dimension idéologique qui, aujourd'hui, est sans doute plus gênante qu'elle ne l'était dans les années 50, période qui a précédé la décolonisation. L'éloge presque inconditionnel des sociétés archaïques et leur description embellie et exaltée seront violemment dénoncés par le Claude Lévi-Strauss de Tristes tropiques (1955). Ce célèbre 
ouvrage critiquera, en montrant l'envers du miroir du travail ethnologique, l'exposé généralement enjolivé de la description idéalisée des sociétés primitives. Mais déjà, on ressent comment cette vision prémonitoire d'un écologisme militant et désespéré au service des civilisations endémiques qu'il faut protéger, au besoin contre elles-mêmes, est ambiguë. Proche des bons sentiments, ne constituera-t-elle pas un alibi et un utile dérivatif vis-à-vis des autres inégalités qui caractérisent les sociétés modernes industrielles et postindustrielles ? Mais, qu'importe l'idéologie, ici secondaire, pour chercher à comprendre quelle est la réelle portée d'un ouvrage comme Apoutsiak. Si cette analyse a un sens dans la perspective communicationnelle qui nous préoccupe, c'est de révéler comment un ouvrage dès lors qu'il est, d'une part, replacé dans son contexte scientifique de production, ou examiné dans ses potentialités de reconnaissance par différentes catégories de lecteurs, échappe aux taxonomies (utiles et nécessaires) constitutives des genres au sein des médias ou des rubriques. Non seulement, comme le remarque Howard S. Becker (1999; 200I), c'est le contexte communicationnel qui oriente la lecture et l'interprétation d'un document authentiquement ethnographique, comparativement à un reportage ou à une œuvre de fiction, non l'économie de sa production. Le classement dans les catégories de la littérature est lui-même largement arbitraire et doit surtout à des impératifs économiques ou commerciaux. Ce qui, en définitive, fait le prix d'un ouvrage comme Apoutsiak, n'est-il pas que tout en assumant, sans faille, sa double fonction d'album pour enfants et de documentaire, il parvienne à demeurer, tout simplement, un chef d'œuvre de littérature à part entière ${ }^{5}$.

\section{Références}

Bakhtine M., 1978, « Le chronotope du roman-idylle », pp. 367-383, in : Bakhtine M., Esthétique et théorie du roman, trad. du russe par D. Olivier, Paris, Gallimard.

Becker H. S., 200I, "Sociologie visuelle, photographie documentaire et photojournalisme », Communications, 7I, pp. 333-352.

Benjamin W., 1972, «Vue perspective sur le livre pour enfants », pp. 93- I08, in : Benjamin W., Je déballe ma bibliothèque. Une pratique de la collection, trad. de l'allemand par P. Ivernel, Paris, Éd. Rivages, 2000.

Bourdieu P., 1972, Esquisse d'une théorie de la pratique, Genève, Droz.

Bresson F., 1981, «Compétence iconique et compétence linguistique », Communications, 33, pp. $185-196$.

\footnotetext{
${ }^{5}$ Ce texte doit beaucoup aux suggestions et remarques de Michéa. Catherine Ruppli m'a aidé patiemment à retrouver les archives et la trace des protagonistes. Pour vérifier les conditions de production, nous avons recueilli des informations de très nombreux informateurs. Merci en particulier à Jean-Christophe, dédicataire de l'ouvrage, pour ses conseils et son soutien.
} 
Chamboredon J.-C., Fabiani J.-L., 1977, « Les albums pour enfants. Le champ de l'édition et les définitions sociales de l'enfance », Actes de la recherche en sciences sociales, pp. 60-79.

Chartier A. M., Hébrard J., 1989, Discours sur la lecture 1880-1980, Paris, Bibliothèque publique d'information du Centre Georges Pompidou.

Chartier R., Martin H. J., 1991, Histoire de l'édition française. Le livre concurrencé : 19001950, vol. 4, Paris, Fayard.

Chevrier J.-F., Roussin P., 200I, dirs, Communications, 7I, « Le parti pris du document. Littérature, photographie, cinéma et architecture au $X X$ e siècle $»$.

Corner J., 1993, « Genres télévisuels et réception », Hermès, II - I2, pp. I 17-124.

Faucheux P., 1978, Écrire l'espace, Paris, R. Laffont.

Genette G., 1987, Seuils, Paris, Éd. du Seuil.

Lévi-Strauss C., 1955, Tristes tropiques, Paris, Plon.

Malaurie J., 1955, Les derniers rois de Thulé. Avec les Esquimaux polaires, face à leur destin, Paris, Plon, 1989.

- 1990, Ultima Thulé. Les Inuit nord-groenlandais face aux conquérants du Pôle (I8181993), Paris, Plon.

— 1999, Hummocks : relief de mémoire, Tome I, Nord Groenland, Arctique central canadien, Paris, Plon.

Mauss M., 1947, Manuel d'ethnographie, Paris, Payot, 2002.

Singly F. de, 1993, « Les jeunes et la lecture », Les dossiers de l'éducation, 24.

Snow C. P., 1959, Les deux cultures, suivies de : Supplément aux deux cultures, trad. de l'anglais par C. Noël, Paris, Pauvert, 1968.

Soriano M., 1968, Les contes de Perrault. Culture savante et traditions populaires, Paris, Gallimard.

Victor P.-É., 1938, Boréal. La joie dans la nuit, illustré de nombreux croquis et de cartes par l'auteur, Paris, Grasset, 1961. 